\title{
Adaptive Strategies for the Elderly in Inhibiting Irrelevant and Conflict No-Go Trials while Performing the Go/No-Go Task
}

\author{
Shulan Hsieh ${ }^{1,2 *}$, Mengyao $\mathrm{Wu}^{1,3}$ and Chien-Hui Tang ${ }^{1}$ \\ ${ }^{1}$ Cognitive Electrophysiology Laboratory: Control, Aging, Sleep, and Emotion (CASE), Department of Psychology, National \\ Cheng Kung University, Tainan, Taiwan, ${ }^{2}$ Institute of Allied Health Sciences, National Cheng Kung University, Tainan, Taiwan, \\ ${ }^{3}$ Department of Occupational Therapy, Shu-Zen Junior College of Medicine and Management, Kaohsiung, Taiwan
}

\section{OPEN ACCESS}

Edited by:

Junfeng Sun,

Shanghai Jiao Tong University, China

Reviewed by:

Noa Fogelson, Universidade da Coruña, Spain Xiangfei Hong,

Philips Research, China

*Correspondence:

Shulan Hsieh

psyhs/@mail.ncku.edu.tw

Received: 20 September 2015 Accepted: 10 December 2015

Published: 06 January 2016

Citation:

Hsieh S, Wu M and Tang C-H (2016) Adaptive Strategies for the Elderly in Inhibiting Irrelevant and Conflict

No-Go Trials while Performing the Go/No-Go Task.

Front. Aging Neurosci. 7:243. doi: 10.3389/fnagi.2015.00243
This study aimed to differentiate whether or not older adults are more prone to distraction or conflict, as induced by irrelevant and conflict no-go stimuli (irNOGO and cfNOGO), respectively. This study also aimed to determine whether or not older adults would devote more effort to withholding a no-go trial in the higher-control demand condition (20\% no-go trials' probability) as compared to the lower-control demand condition (50 and $80 \%$ no-go trials' probability). A total of 96 individuals were recruited, and each of the three no-go trials' probability conditions included 32 participants (16 younger adults and 16 older adults). Both behavioral and event-related potential (ERP) data were measured. The behavioral results showed that the older adults performed more poorly than the younger adults for the go trials, as reflected by slower reaction times (RTs) and higher numbers of omission errors in the go trials. In contrast, in the no-go trials, the older adults counter-intuitively exhibited similar behavioral performance (i.e., equivalent commission errors) as compared to the younger adults. The ERP data further showed that the older adults (but not the younger adults) exhibited larger P3 peak amplitudes for the irNOGO than cfNOGO trials. Yet, on the other hand, the older adults performed more poorly (i.e., had more commission errors) in the cfNOGO than irNOGO trials. These results seem to suggest that the older adults recruited more control processes in order to conquer the commitment of responses in the no-go trials, especially in the irNOGO trials. This age-related compensatory response of recruiting more control processes was specifically seen in the $20 \%$ no-go trial probability condition. This study therefore provides a deeper understanding into how older adults adopt strategies for performing the go/no-go task such as devoting more control processes to inhibiting the irNOGO trials compared to the cfNOGO trials in order to cope with their deficient inhibition ability.

Keywords: event-related potential, distraction, inhibition, no-go, probability

\section{INTRODUCTION}

Daily life often requires an individual to produce a certain behavior in an environment that is filled with irrelevant and often distracting information. Hence, to achieve the goal of acting adequately, one has to adaptively overcome the competition brought by the strong, yet inappropriate, momentary tendency to inhibit a prepotent yet unintentional response in order 
to prevent negative consequences. These adaptive processes are broadly termed inhibition or suppression, and they have often been hypothesized to involve the frontal lobe functions (e.g., Rogers et al., 1998). Given the importance of inhibition in everyday life, several studies have attempted to address the issue of whether or not elderly people could successfully cope with brain degeneration, particularly in the frontal lobes (Dempster, 1992; Raz, 2000; West, 2000; Dennis and Cabeza, 2008), to achieve the goal of acting adequately in everyday life scenarios.

However, the findings regarding how capable an elderly person can be in performing an inhibition-related task are equivocal: some studies showed that the elderly suffered from generic inhibition deficit, hence, they were incapable of performing any inhibition-related task as compared to the younger adults (Hasher and Zacks, 1988; Bokura et al., 2002; Vallesi et al., 2009; Vallesi, 2011; Lucci et al., 2013; Pires et al., 2014), while some other studies have shown that the elderly could develop some strategies to compensate for their deficiency in achieving the task goal (Cabeza et al., 2002; Phillips and Andrés, 2010; Hsieh and Fang, 2012; Hsieh et al., 2012; Hsieh and Lin, 2014). Many factors such as different experimental settings, different populations, and different types of stimuli and requirements could potentially contribute to the discrepancies. Among these factors, we suggest that task parameters may play a critical role in older adults' performance strategies. Hence, in this study we manipulated two task parameters in a go/no-go task paradigm to address the inhibition proficiency of older adults. We focused specifically on a go/no-go task paradigm because such an inhibition task has been proven to be sensitive to aging (Bokura et al., 2002; Vallesi et al., 2009; Vallesi, 2011; Lucci et al., 2013; Pires et al., 2014). The two-task parameters we manipulated here include a no-go stimulus and a no-go stimulus probability. With regard to the type of no-go stimulus, we incorporated two types of no-go stimuli: irrelevant no-go (irNOGO) and conflict no-go (cfNOGO). The irNOGO stimuli belong to a different semantic category from that of the go stimuli (e.g., numbers vs. letters), which yields an obvious distinction between the target (go) and non-target (no-go) stimuli, even though the no-go stimuli may share a common feature with the go stimuli. For example, both the irNOGO and go stimuli could be either red or blue, but they belong to numbers and letters, respectively. In contrast, the cfNOGO stimuli share the same category (i.e., letters) as the go stimuli. The rationale for this manipulation is that the irNOGO stimuli should be easier to distinguish from the go stimuli; therefore, it is easier inhibit compared to the cfNOGO stimuli. On the other hand, some prior studies in the memory research domain have suggested that older adults were more vulnerable to internal distraction and weakening concentration skills due to their subtle changes in brain activity (e.g., Grady et al., 2006). It is therefore interesting to examine whether or not older adults are also susceptible to external distractions, such as those induced by the irNOGO stimuli in a go/no-go task.

With regard to no-go stimulus probability, we manipulated the probability of no-go trials in a block to be either 20, 50, or $80 \%$. The rationale for the probability manipulation is that with a strong response bias towards go stimuli (20\% no-go probability), one can maximize the engagement of executive control to inhibit no-go stimuli (Bruin and Wijers, 2002; Ford et al., 2004). Subsequently, the performance of a go/no-go task may be more sensitive to aging in the condition of this higher demand condition (20\% of no-go probability).

With the manipulation of these two task parameters, we could examine whether or not aging results in a selective deficit in which task parameters are sensitive to the effect of aging in only some scenarios. Because no overt response can be recorded for no-go trials in a go/no-go paradigm, a neural imaging approach is needed to uncover the underlying processes of the no-go trials. In this study, we employed the event-related potential (ERP) method, which yields higher temporal resolution and uncovers the underlying neural activity for no-go trials.

Two ERP components are considered in this study that have been demonstrated to be sensitive to a go/no-go task, that is, the stimulus-locked P3 and N2 components (for a review, see Pires et al., 2014). Stimuli that trigger a tendency to make incorrect prepotent responses (e.g., incongruent or no-go stimuli) have been found to be associated with enhanced fronto-central N2 amplitude, which is thought to reflect inhibition (Van Boxtel et al., 2001; Roche et al., 2005) and/or response conflict control processes (Falkenstein et al., 2001; Van Veen and Carter, 2002a,b; Nieuwenhuis et al., 2003). Furthermore, Nieuwenhuis et al. (2003) observed that nogo $\mathrm{N} 2$ amplitudes were higher in the high go-prepotency (20\% no-go) condition compared with medium (50\%) and low go-prepotency ( $80 \%$ no-go) conditions, which supports the conflict theory of N2. Following the N2, there is a positive ERP component that peaks at approximately 250-500 ms for both go (known as the go P3) and no-go (known as the no-go P3) trials. Both go and no-go P3s may reflect context updating (Donchin and Coles, 1988), which is necessary for successful ongoing execution and inhibition of prepotency responses. Specifically, the no-go P3 has been shown to be closely related to inhibition (Roberts et al., 1994; Fallgatter and Strik, 1999; Tekok-Kilic et al., 2001; Smith et al., 2008). Hence, by observing how aging may modulate the no-go N2 and P3 components, we can uncover age-related inhibition and/or conflict processes.

\section{MATERIALS AND METHODS}

\section{Participants}

A total of 96 individuals were recruited through the internet and local community advertisements; each of the three no-go probability condition blocks included 32 participants (16 younger adults and 16 older adults). For the $20 \%$ no-go probability condition, 16 young adults ( 9 females) had a mean age of $21.31 \pm 1.40$ years (range 20-25 years) and an average of $15.69 \pm 1.25$ years of education; the 16 elderly adults ( 9 females) had a mean age of $66.38 \pm 4.57$ years (range 61-72 years) and an average of $12.69 \pm 2.15$ years of education. For the $50 \%$ no-go probability condition, the 16 young adults ( 8 females) had a mean 
age of $21.31 \pm 1.30$ years (range 20-25 years) and an average of $15.00 \pm 1.10$ years of education; the 16 elderly adults ( 8 females) had a mean age of $67.25 \pm 4.74$ years (range $60-76$ years) and an average of $12.56 \pm 3.60$ years of education. For the $80 \%$ no-go probability condition, the 16 young adults ( 9 females) had a mean age of $21.63 \pm 1.54$ years (range 19-24 years) and an average of $15.44 \pm 1.21$ years of education; the 16 elderly adults ( 9 females) had a mean age of $68.63 \pm 6.08$ years (range $61-80$ years) and an average of $13.31 \pm 2.75$ years of education (see Table 1 ).

The 2-way analysis of variance (ANOVAs) on age with two between-subjects factors of age and no-go probability showed a significant main effect of age (young: $21.42 \pm$ 1.38 years vs. old: $67.42 \pm 5.09$ years, $F_{(1,90)}=3529.05$, $p<0.01$ ), but it did not show a significant main effect of no-go probability or a significant interaction between age and no-go probability (i.e., all $p s>0.05$ ). The 2-way ANOVA on years of education showed a significant effect of age (young: $12.85 \pm 2.82$ years vs. old: $15.38 \pm 1.18$ years, $F_{(1,90)}=31.18$, $p<0.01)$. No significant effect of no-go probability or interaction between age and no-go probability was found (all ps $>0.05)$.

All participants provided their written informed consent, and the study protocol was approved by the Institutional Review Board (IRB) of the National Cheng Kung University Hospital, Taiwan. All participants were paid NT \$500-1000 (US \$15-30) for approximately $3 \mathrm{~h}$ of participation. All participants were right-handed, free of neurological and psychological disorders, and had normal or corrected-to-normal vision. The Mini-Mental State Examination (MMSE; Folstein et al., 1975) screened all participants for dementia based on the following screening criteria: $25-30$ points = normal; $21-24$ points = mild dementia; $14-20$ points = moderate dementia; and $\leq 13$ points $=$ severe dementia. For the $20 \%$ no-go probability condition, the mean MMSE score was $28.63 \pm$ 0.60 for the younger adults and $27.06 \pm 1.14$ for the older adults. For the $50 \%$ no-go probability condition, the mean MMSE score was $28.50 \pm 0.71$ for the younger adults and $26.68 \pm 1.10$ for the older adults. For the $80 \%$ no-go probability condition, the mean MMSE score was $28.18 \pm$ 1.01 for the younger adults and $27.19 \pm 0.73$ for the older adults.

The 2-way ANOVA on the MMSE score showed a significant effect of age (young: $26.98 \pm 1.03$ vs. old: $28.44 \pm 0.81$, $\left.F_{(1,90)}=58.15, p<0.01\right)$ but no significant effect of no-go probability; also, there was no significant interaction between age and no-go probability (all $p s>0.05$ ). Since there was a significant effect of age on MMSE scores, this may be a potential confounding variable for the results, i.e., the changes may be due to cognitive decline rather than physiological aging. In order to preclude the possible contribution of cognitive decline in the older group, we have additionally run an analysis of covariance (ANCOVA) using MMSE as a covariate factor. The results of the ANCOVA showed the same patterns as those of the ANOVA reported here. Hence, although the older adults exhibited lower MMSE scores, they did not confound the current findings.

\section{Stimuli}

The stimuli were generated using E-Prime software (Psychology Software Tools, Inc., Pittsburgh, PA, USA) and were presented in red or blue against a black background on the center of a computer screen that was placed at a distance of $90 \mathrm{~cm}$ from the participant. In this task, go stimuli were red or blue vowels ("A", "E", "I", "U”) and no-go stimuli were either red or blue consonants ("L", "N", "P", “Z”; designed as cfNOGO stimuli) or red and blue numbers (“3”, " 4 ", “5”, “6”; designed as irNOGO stimuli).

\section{Design and Procedure}

Each trial began with the presentation of a white fixation cross "+" for a duration of $200 \mathrm{~ms}$, followed by a go or no-go stimulus for a duration of $300 \mathrm{~ms}$. This was then replaced by a black blank screen that awaited a response or until $1800 \mathrm{~ms}$ elapsed if no response was recorded. Also, there was an additional waiting duration that varied randomly between 1-1000 ms before the next trial commenced (see Figure 1). Participants were required to make (for a go stimulus) or withhold (for a no-go stimulus) a response as soon and as accurately as possible.

The total number of trials in the no-go probability condition block was 960 . The probabilities of no-go stimuli were 20\% (192 trials), 50\% (480 trials), and 80\% (768 trials), respectively. Among the no-go trials, cfNOGO and irNOGO stimuli comprised $50 \%$ each. There were at least 36 practice trials (more trials if necessary) before each condition block. During the practice session, feedback of "correct" or "incorrect" was given at the end of each trial

TABLE 1 | The demographic information for all participants in all conditions.

\begin{tabular}{|c|c|c|c|c|c|c|}
\hline & \multicolumn{2}{|c|}{$20 \%$ no-go } & \multicolumn{2}{|c|}{$50 \%$ no-go } & \multicolumn{2}{|c|}{$80 \%$ no-go } \\
\hline & Younger & Older & Younger & Older & Younger & Older \\
\hline Gender (male/female) & $7 / 9$ & 7/9 & $8 / 8$ & $8 / 8$ & $7 / 9$ & $7 / 9$ \\
\hline Age (year) & $21.31(1.36)$ & $66.38(4.43)$ & $21.31(1.26)$ & $67.25(4.59)$ & $21.63(1.49)$ & $68.63(5.88)$ \\
\hline Education (year) & $15.69(1.21)$ & 12.69 (2.08) & $15.00(1.06)$ & $12.56(3.48)$ & $15.44(1.17)$ & $13.31(2.66)$ \\
\hline $\mathrm{BDI}$ & $3.44(2.06)$ & $5.25(4.71)$ & 4.69 (3.58) & 6.69 (3.58) & $3.69(2.17)$ & $4.25(3.44)$ \\
\hline MMSE & $28.63(0.60)$ & $27.06(1.14)$ & $28.50(0.71)$ & $26.69(1.10)$ & $28.19(1.01)$ & $27.19(0.73)$ \\
\hline
\end{tabular}

BDI, Beck Depression Inventory; MMSE, Mini-Mental State Examination; the value in each cell denotes the mean, and the value in each bracket denotes standard deviation. 


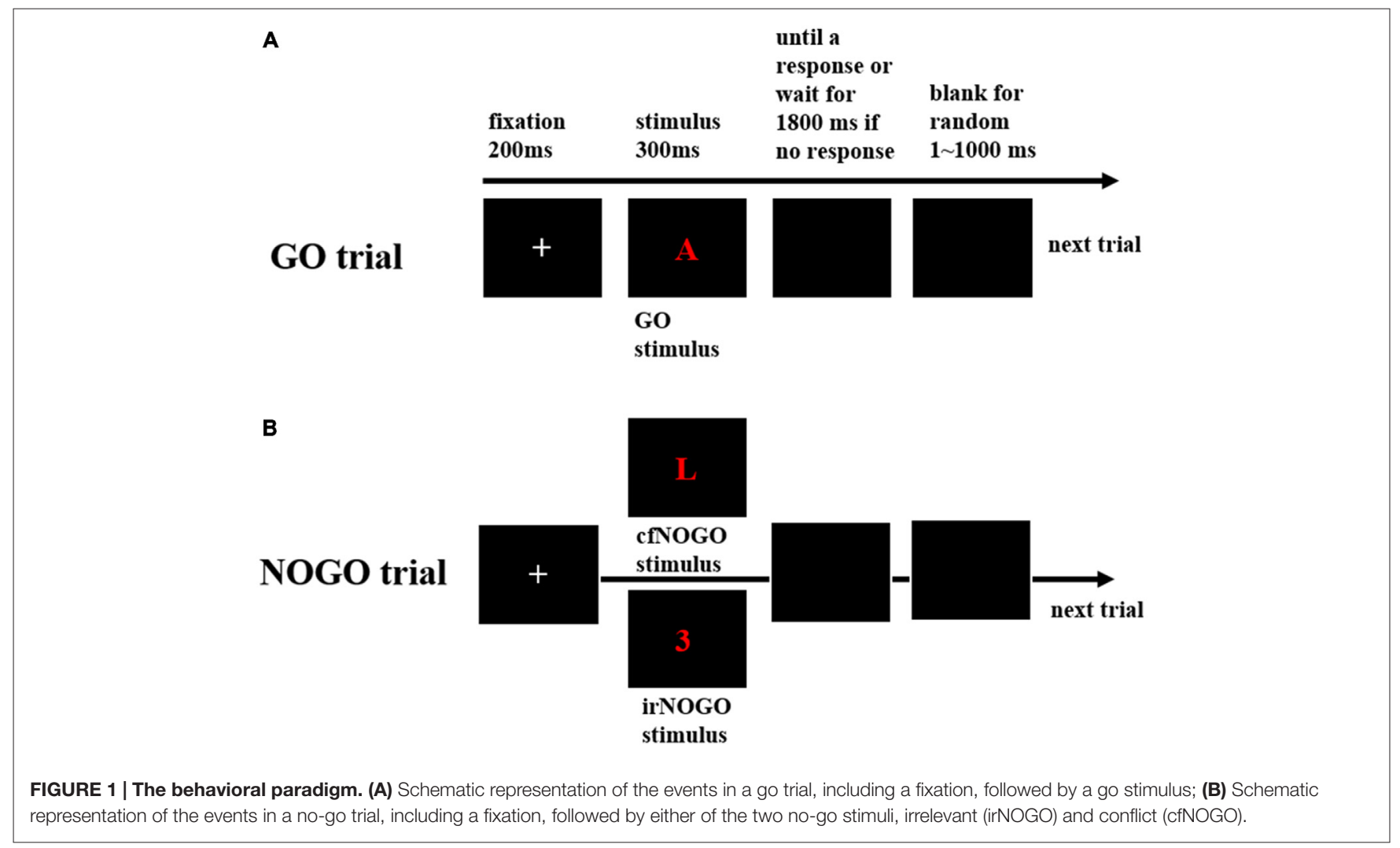

to facilitate the participants' familiarization with the response rule.

\section{EEG Recording}

The participants were seated in a comfortable chair in a soundattenuated room during the experiment. Electroencephalography (EEG) activity was continuously recorded using Neuroscan SynAmp2 amplifier and Q-Cap (Neuroscan Q-Cap: AgCl-32 electrode cap; Neuroscan, Inc., El Paso, TX, USA) from 32 scalp electrodes. The vertical electrooculogram (EOG) was recorded by two electrodes $2 \mathrm{~cm}$ above and $2 \mathrm{~cm}$ below the left eye, and the horizontal EOG was recorded by two electrodes $1 \mathrm{~cm}$ external to the outer canthus of each eye. A ground electrode was placed on the forehead. The electrodes were initially referenced online to the left mastoid and offline to the average of the left and right mastoids. Electrode impedances were maintained below $5 \mathrm{k} \Omega$. The EEG and EOG signals were amplified and digitized at a sampling rate of $500 \mathrm{~Hz}$, with an online high-pass filter of $0.1 \mathrm{~Hz}$ and a low-pass filter of $30 \mathrm{~Hz}$. Ocular artifacts associated with blinks were corrected by the ocular reduction command offered by the Neuroscan software (Neuroscan, Inc., El Paso, TX, USA) and were then further removed via an algorithm (Neuroscan software) that rejected any epoch if the signal was below $-50 \pm 50 \mu \mathrm{V}$, if the drift of the EEG from baseline exceeded $-50 \pm 50 \mu \mathrm{V}$, or if the A/D converts became saturated. The total rejection rate across the various conditions averaged approximately 23,28 and $24 \%$ for each no-go probability condition block, respectively.

\section{Event-Related Potential (ERP) Analysis}

We focused on the N2 and P3 components as they have been hypothesized to link inhibition and response conflict control processes. Moreover, because go and no-go trials involve different processes with respect to motor execution, we separated the ERP analyses of go trials from those of no-go trials. In each condition block, stimulus-locked epochs were taken from the continuous EEG signal and time-locked to the onset of the go/no-go stimulus from $-50 \mathrm{~ms}$ to $800 \mathrm{~ms}$ for all recording channels. For each channel, all stimulus-locked epochs were baseline-corrected by obtaining the mean level of activity in the period from $50 \mathrm{~ms}$ before to $50 \mathrm{~ms}$ after target onset and then subtracting that average from the level of activity at the sample point. The N2 was found to be maximal at the $\mathrm{FCz}$ site, whereas the P3 was maximal at the $\mathrm{Cz}$ site in this study. Hence, we searched for the peak-topeak amplitude at the $\mathrm{FCz}$ site during the time windows of 150-350 $\mathrm{ms}$ (positive dip) and 250-450 ms (negative peak) following the onset of the stimulus and then computed the voltage difference between the positive dip and negative peak as the stimulus-locked N2 peak-to-peak amplitude. Then, we searched for the peak latency and peak amplitude at the $\mathrm{Cz}$ site during the time window of $300-750 \mathrm{~ms}$ following the onset of the stimulus and then computed the voltage difference between the peak amplitude relative to the baseline as the stimulus-locked P3 peak amplitude and the time point at which the P3 peak amplitude occurred as the P3 peak latency. 


\section{Statistical Analysis}

The 2-way ANOVAs for the go trials were performed on the behavioral and ERP data, respectively, with two between-subject factors of age and no-go probability. The 3-way ANOVAs for the no-go trials were performed on the behavioral and ERP data, respectively, with two between-subjects factors of age and nogo probability and one within-subject factor of no-go stimulus types. Post hoc analysis following the significant effect of nogo probability (with three levels) was performed using Tukey tests. When two (or more) factors in the ANOVA showed a statistically significant interaction, we carried on analyzing the simple main effects which involve the examination of the effect of one factor at one level of the other factor. That is, the data were split for each level of one factor and one-way ANOVAs were conducted. Like any other one-way ANOVA with more than two levels, after the significant F, a post hoc Tukey test was conducted to find out which pair (or pairs) of means was (were) statistically different. To overcome the inflation of Type 1 error when a series of simple main effect analyses were conducted, we used the Bonferroni correction to adjust the $p$ value. In addition, in the choice of error term for simple main effect test, we followed the pooled error term approach advised by Howell (2010). That is, Mean Square Error (MSE) from the original factorial ANOVA was used as opposed to MSE from the followup one-way ANOVA in calculating the F for the simple main effects. The detailed steps for the calculation are described in Howell's (2010) book (e.g., ps. 483-488 in his book, "Statistical Methods for Psychology").

\section{RESULTS}

\section{Behavioral Data Analysis}

The first trial of each block and trials with RTs faster than $150 \mathrm{~ms}$ or slower than $1500 \mathrm{~ms}$ were discarded from further analysis. The behavioral results are shown in Figure 2, including the go trials' RT (Figure 2A) and omission rates (Figure 2B) and the no-go trials' commission errors (Figure 2C).

\section{Reaction Time (RT) on Go Trials}

The 2-way ANOVA on the RTs of the go trials showed that the younger adults generally performed faster than the older adults, $F_{(1,90)}=63.11, p<0.001$, and that RTs were faster in the $20 \%$ no-go probability (high go-prepotency) and the $50 \%$ no-go probability (medium go-prepotency) conditions than in the $80 \%$ no-go probability (low go-prepotency) condition (no-go probability, $F_{(2,90)}=21.42, p<0.001$; all Tukey tests: $\left.p s<0.005\right)$. There was no significant interaction between the two factors, $F_{(2,90)}=2.61, p=0.08$.

\section{Percentage of Error (PE) on Go Trials: Omission Errors}

The 2-way ANOVA on the omission errors of the go trials showed that the younger adults generally performed better (fewer omission errors) than the older adults, $F_{(1,90)}=15.20, p<0.001$, and that omission errors were fewer in the 20 and $50 \%$ no-go probability conditions (3.46 and 3.04\%) than in the $80 \%$ no-go probability condition $\left(7.70 \% ; F_{(2,90)}=4.92, p<0.01\right.$; all Tukey tests: $p<0.05)$. There was no significant interaction between the two factors, $F_{(2,90)}=0.93, p=0.40$.

\section{Percentage of Error (PE) on No-Go Trials: Commission Errors}

The 3-way ANOVA on the commission errors of the nogo trials showed that there was no significant effect of age, $F_{(1,90)}=2.14, p=0.15$, but there was a significant effect of no-go probability, $F_{(2,90)}=47.41, p<0.0001$. Commission errors were more frequent in the $20 \%$ no-go probability condition $(16.53 \%)$ than in the $50 \%(4.13 \%)$ and $80 \%$ no-go probability conditions (2.95\%; all Tukey tests: $p s<0.05$ ). There was also a significant effect of no-go stimulus type, $F_{(1,90)}=88.31, p<0.0001$, showing that irNOGO $(4.78 \%)$ stimuli elicited fewer commission errors than cfNOGO (10.96\%) stimuli. There was also a significant 2 -way interaction between no-go probability and no-go stimulus type, $F_{(2,90)}=26.52$, $p<0.0001$. Follow-up tests for this interaction showed that the effect of no-go probability was significant on both cfNOGO trials $\left(F_{(2,90)}=71.51, p<0.0001\right)$ and irNOGO trials $\left(F_{(2,90)}=14.41\right.$, $p<0.001)$, yet there was a larger effect on the cfNOGO trial type. In addition, the simple effect of the no-go stimulus type (fewer commission errors for the irNOGO than cfNOGO trials) was significant only in the 20 and $50 \%$ no-go probability conditions.

\section{Summary of Behavioral Data}

To summarize, the older adults appeared to perform more poorly than the younger adults on go trials, as reflected by their slower go RTs and higher go omission errors; this age effect was not modulated by no-go probability. In contrast, the older adults counter-intuitively exhibited similar behavioral performance (i.e., equivalent commission errors) as compared to the younger adults. In addition, it appeared to be more difficult for both age groups to withhold a response to cfNOGO stimuli compared to irNOGO stimuli, based on the data of commission errors.

\section{Event-Related Potential (ERP) Data}

ERPs associated with each condition and age group at the $\mathrm{Fz}$, $\mathrm{FCz}, \mathrm{Cz}, \mathrm{CPz}$, and $\mathrm{Pz}$ sites as well as topographic maps are shown in Figures 3A,B for go trials and Figures 4A,B for no-go trials.

\section{P3 Peak Latency on Go Trials}

The 2-way ANOVA on the P3 peak latencies of go trials showed that the younger adults exhibited earlier P3 peak latencies than the older adults (young: $526.04 \mathrm{~ms}$ vs. old: $596.75 \mathrm{~ms}$ ), $F_{(1,90)}=26.63, p<0.0001$, but there was no significant effect of no-go probability, $F_{(2,90)}=0.81, p=0.45$. There was also no significant 2-way interaction between age and no-go probability, $F_{(2,90)}=1.11, p=0.34$ (see Figure 5A). 


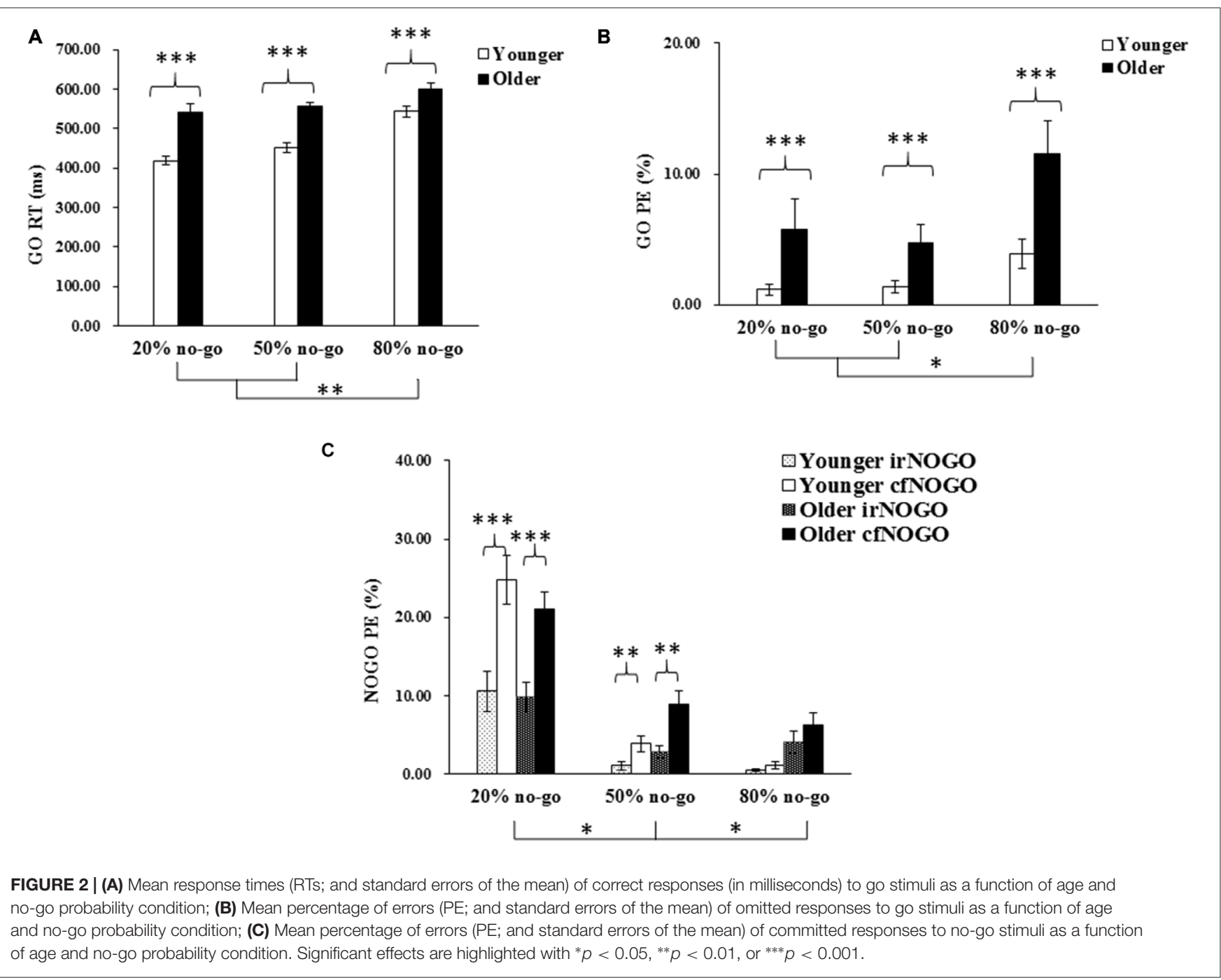

\section{P3 Peak Amplitude on Go Trials}

The 2-way ANOVA on the P3 peak amplitudes of the go trials showed larger P3 peak amplitudes for the younger $(12.53 \mu \mathrm{V})$ than older $(7.01 \mu \mathrm{V})$ adults, $F_{(1,90)}=34.58, p<0.0001$, but no significant main effect of no-go probability, $F_{(2,90)}=0.79$, $p=0.46$. Yet, there was a significant 2-way interaction between age and no-go probability, $F_{(2,90)}=4.94, p<0.01$. A simple effect test following this interaction showed that the significant main effect of no-go probability occurred only for the younger adults, $F_{(2,90)}=4.80, p<0.05$, but not for the older adults, $F_{(2,90)}=0.93$, $p=0.40$, and that the significant effect of age occurred in the $50 \%$, $F_{(1,90)}=11.49, p<0.005$, and $80 \%, F_{(1,90)}=31.59, p<0.0001$, nogo probability conditions but not in the $20 \%$ no-go probability conditions, $F_{(1,90)}=1.38, p=0.24$ (see Figure 5B).

\section{P3 Peak Latency on No-Go Trials}

The 3-way ANOVA on the P3 peak latencies of no-go trials showed that the younger adults exhibited earlier $\mathrm{P} 3$ peak latencies than the older adults (young: $516.35 \mathrm{~ms}$ vs. old: $641.46 \mathrm{~ms}$ ), $F_{(1,90)}=162.85, p<0.001$, and that irNOGO stimuli elicited earlier P3 peak latencies than cfNOGO stimuli, $F_{(1,90)}=30.42$, $p<0.0001$. There were no significant effect of no-go probability and any interactions (age $\times$ type of no-go stimulus: $F_{(1,90)}=2.03$, $p=0.16$; no-go probability $\times$ type of no-go stimulus: $F_{(2,90)}=0.08, p=0.93$; age $\times$ type of no-go stimulus $\times$ no-go probability: $F_{(2,90)}=0.922, p=0.40$, see Figure 6A.

\section{P3 Peak Amplitude on No-Go Trials}

The 3-way ANOVA on the P3 peak amplitudes of the no-go stimuli showed that $\mathrm{P} 3$ peak amplitudes for the no-go trials were larger in the $20 \%$ no-go probability condition than in the 50 and $80 \%$ no-go probability conditions, $F_{(2,90)}=15.58, p<0.0001$ (all Tukey tests: $p s<0.01$ ). There was also a significant 2-way interaction between age and no-go stimulus type, $F_{(1,90)}=13.49$, $p<0.001$.

A simple effect test on the interaction between age and no-go stimulus type showed that only older adults exhibited larger P3 peak amplitudes for irNOGO stimuli than for cfNOGO stimuli but not for the younger adults (young: $F_{(1,90)}=1.80, p=0.19$; old: $F_{(1,90)}=14.83, p<0.001$; see Figure 6B). 

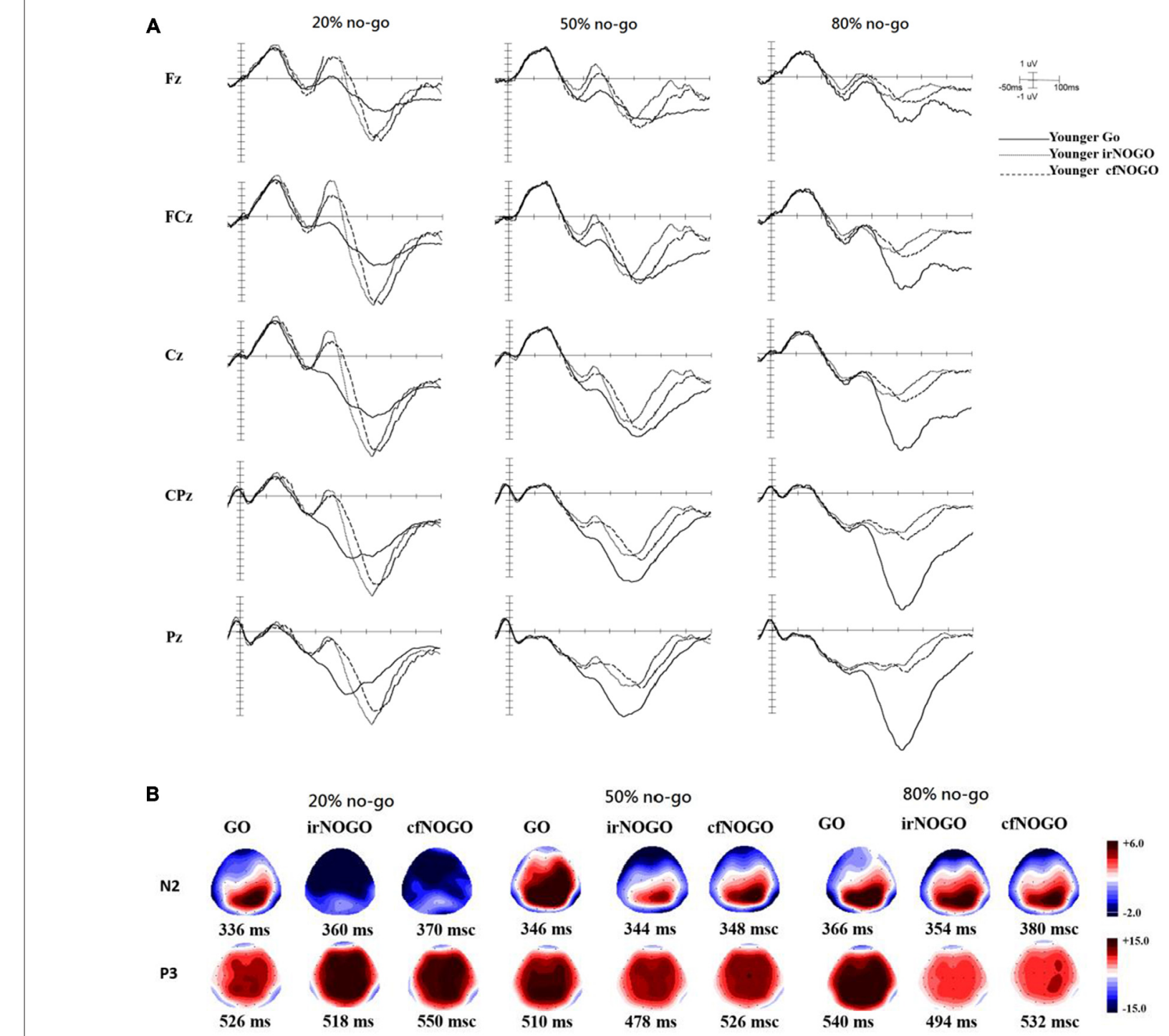

FIGURE 3 | (A) ERP waveforms as a function of no/no-go condition (GO, irNOGO, cfNOGO) and no-go probability condition at five representative electrodes of Fz, FCz, Cz, CPz, and Pz for the younger adults. (B) Topographic maps of the GO N2, irNOGO N2, cfNOGO N2, GO P3, irNOGO P3, and cfNOGO P3, separately for each no-go probability condition and for the younger adults. Each map describes the topographic distribution at the peak latency for each condition.

\section{Summary of the P3 Findings}

\section{Go P3 Peak Latency and Amplitude}

For the go P3 peak latency, there was a significant effect of age that showed an earlier P3 peak latency for the younger adults. As for the go P3 peak amplitude, the significant effect of aging (younger larger than older) occurred only in the 50 and $80 \%$ no-go probability conditions. In addition, the significant effect of no-go probability occurred only for the younger adults, i.e., showing smaller go P3 peak amplitudes in the $20 \%$ than $80 \%$ no-go probability condition but not for the older adults.

\section{No-Go P3 Peak Latency and Amplitude}

The significant effect of age was found for the overall no-go P3 peak latency. In addition, both age groups exhibited earlier
P3 peak latencies for the irNOGO trials compared to the cfNOGO trials. As for the no-go P3 peak amplitude, there was a significant effect of no-go probability for both age groups, showing larger no-go P3 peak amplitudes in the $20 \%$ no-go probability conditions compared to the 50 and $80 \%$ no-go probability conditions. More importantly, only the older adults exhibited larger P3 peak amplitudes for the irNOGO trials compared to the cfNOGO trials. These results suggested that while the younger adults exhibited similar no-go P3 peak amplitudes between the irNOGO and cfNOGO trials, the older adults exhibited more prominent P3 peak amplitudes for the irNOGO than cfNOGO trials. Subsequently, this phenomenon resulted in a significant effect of age for the cfNOGO trials but not for the irNOGO trials. 
A

A
Fz<smiles>CCCC=CCCCC</smiles>

FCz<smiles>CCCC=CC1=CC=CC1</smiles>

$\mathrm{Cz}$<smiles>C=CC(C=CCCC)=CCC</smiles>

$\mathrm{CP}$ z

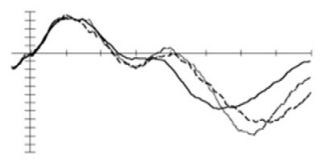

Pz<smiles>C#CCCCCCCC</smiles>

B

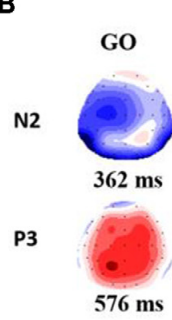

\section{$20 \%$ no-go

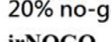

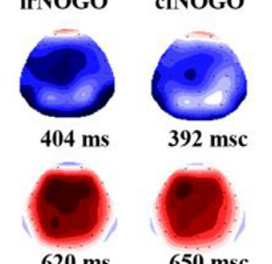
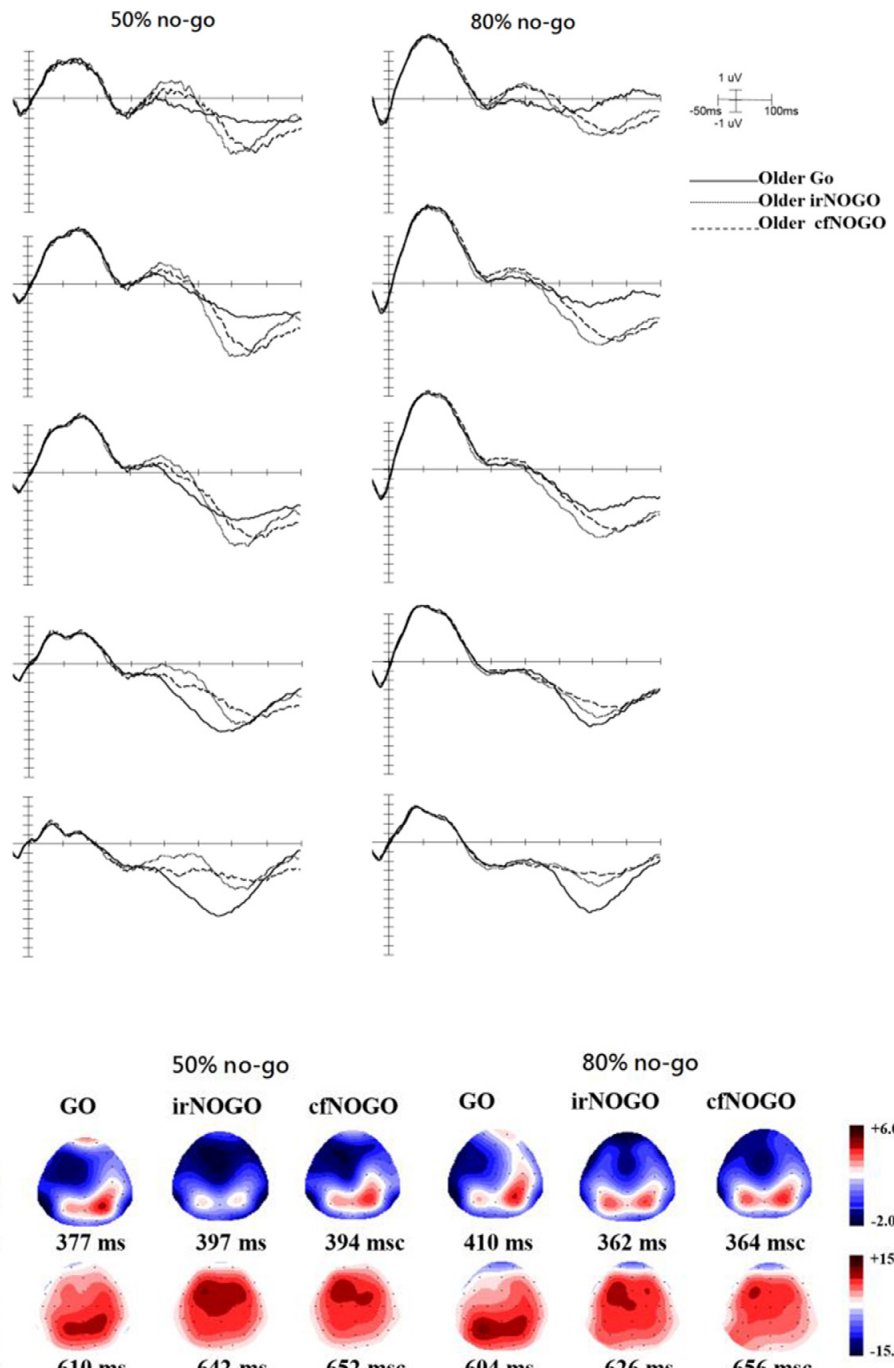

$50 \%$ no-go
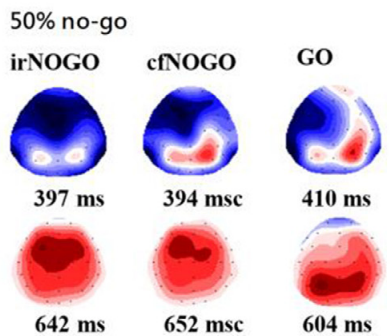

$80 \%$ no-go

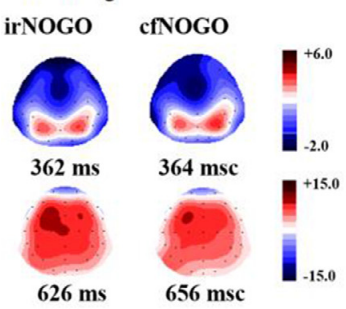

FIGURE 4 | (A) ERP waveforms as a function of no/no-go condition (GO, irNOGO, cfNOGO) and no-go probability condition at five representative electrodes of Fz, FCz, Cz, CPz, and Pz for the older adults. (B) Topographic maps of the GO N2, irNOGO N2, cfNOGO N2, GO P3, irNOGO P3, and cfNOGO P3, separately for each no-go probability condition and for the older adults. Each map describes the topographic distribution at the peak latency for each condition.

\section{N2 Peak-to-Peak Amplitude on Go Trials}

The 2-way ANOVA on the N2 peak-to-peak amplitudes of the go trials showed no significant effects or interactions (age: $F_{(1,90)}=1.24, p=0.27$; no-go probability: $F_{(2,90)}=1.35, p=0.27$; age $x$ no-go probability: $F_{(2,90)}=1.89, p=0.19$; see Figure 7$)$.

\section{N2 Peak-to-Peak Amplitude on No-Go Trials}

The 3-way ANOVA on the N2 peak-to-peak amplitudes of no-go stimuli showed that the younger adults exhibited larger $\mathrm{N} 2$ peak-to-peak amplitudes $(-5.97 \mu \mathrm{V})$ than the older adults $(-4.52 \mu \mathrm{V}), F_{(1,90)}=8.24, p<0.01$. Also, N2 peak-to-peak amplitudes were larger in the $20 \%$ no-go probability condition compared to the $50 \%$ no-go probability condition and amplitudes in the latter condition were larger than amplitudes in the $80 \%$ no-go probability condition, $F_{(2,90)}=33.87, p<0.0001$ (all Tukey tests: $p s<0.01$ ). There was also a significant effect of no-go stimulus type, $F_{(1,90)}=7.61, p<0.01$; this indicates that irNOGO stimuli elicited larger N2 peak-to-peak amplitudes than cfNOGO stimuli. There were significant 2 -way interactions between age and no-go probability, $F_{(2,90)}=3.78, p<0.05$, and between no-go probability and no-go stimulus type, $F_{(2,90)}=7.34$, $p<0.005$.

The simple effect test for the interaction between age and no-go probability showed that the effect of age was only significant for the $20 \%$ no-go probability condition, where the younger adults exhibited larger N2 peak-to-peak amplitudes than older adults. On the other hand, both age groups exhibited significant effects for the no-go probability on the N2 peak-topeak amplitude. 


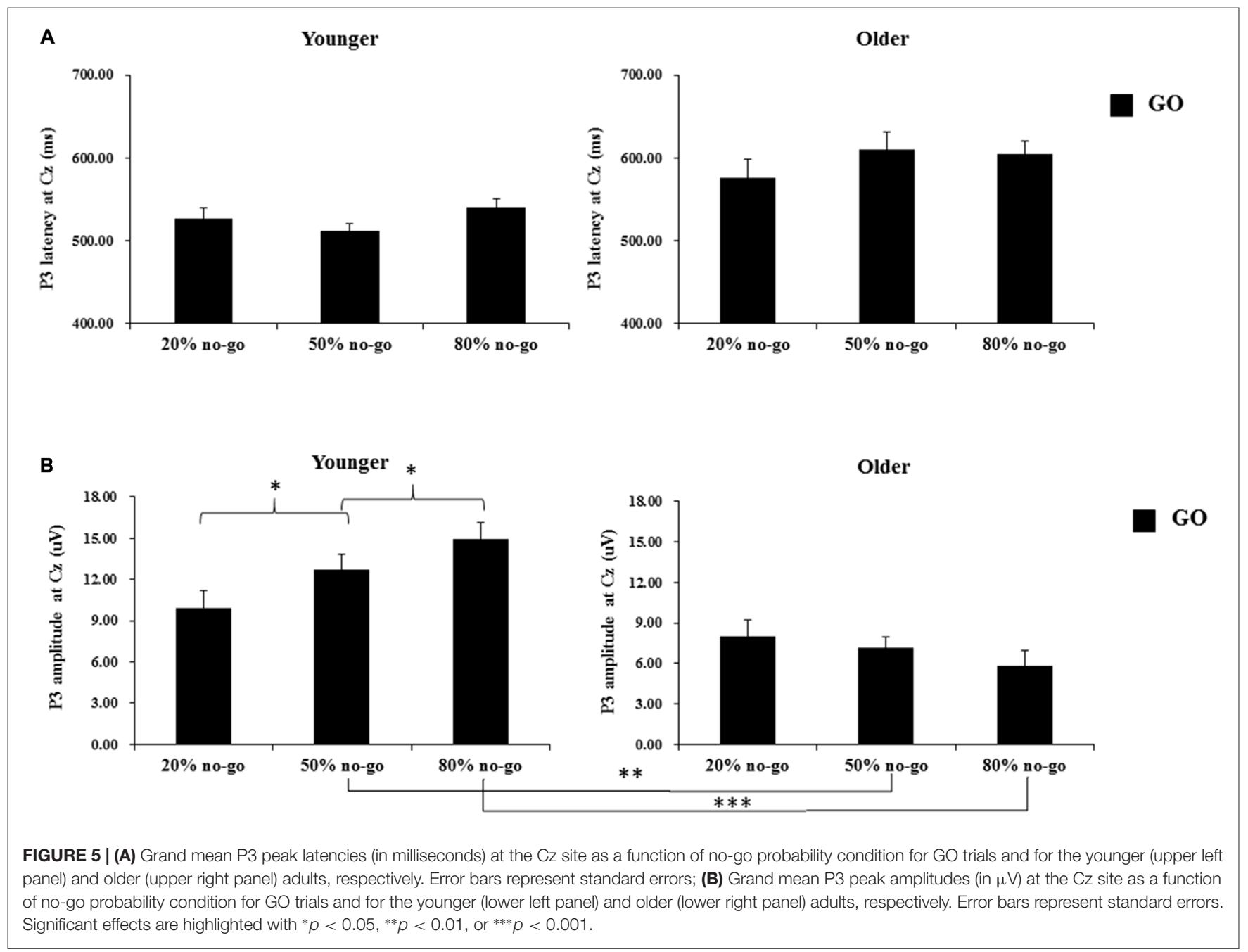

The simple effect test on the interaction of no-go probability and no-go stimulus type nevertheless showed a significant main effect of no-go stimulus type (i.e., the irNOGO trials elicited larger N2 amplitudes than cfNOGO trials) in the $20 \%$ no-go probability condition, $F_{(1,90)}=20.03, p<0.0001$, but not for the 50 and $80 \%$ no-go probability condition $\left(50 \%: F_{(1,90)}=1.46\right.$, $\left.p=0.23 ; 80 \%: F_{(1,90)}=0.81, p=0.37\right)$. Furthermore, for both the irNOGO and cfNOGO stimuli, the amplitudes were larger in the $20 \%$ no-go probability condition than in the 50 and $80 \%$ no-go probability condition (all $p s<0.05$; see Figure 8).

\section{Summary of the N2 Findings}

\section{Go N2 Peak-to-Peak Amplitude}

The significant effect of no-go probability on go N2s occurred only for the younger adults and between the 50 and $80 \%$ no-go probability conditions, whereas none of the significant main effects and interactions were found for the older adults.

\section{No-Go N2 Peak-to-Peak Amplitude}

The significant effect of age (i.e., the younger adults exhibited larger N2 amplitudes than the older adults) on no-go N2s occurred mainly in the $20 \%$ no-go probability condition. In addition, the significant effect of no-go trial type (i.e., irNOGO trials exhibited larger N2 amplitudes than cfNOGO trials) was found only for the $20 \%$ no-go probability condition for both age groups. Furthermore, for both the irNOGO and cfNOGO stimuli, the amplitudes were larger in the $20 \%$ no-go probability condition than in the 50 and $80 \%$ no-go probability conditions.

\section{DISCUSSIONS}

The aim of this study was to evaluate whether older adults exhibited selective inhibition deficit by using a go/no-go paradigm with the manipulation of no-go stimulus-type and no-go probability. The current behavioral results showed that the older adults performed more poorly than the younger adults as reflected from their slower RTs and their higher omission errors in the go trials. This age difference was not further modulated by no-go probability, despite the fact that there was a significant effect of no-go probability on both RTs and omission errors. Interestingly, in contrast to the inferior performance on go trials for the older adults compared to the younger adults, the older adults counter-intuitively exhibited similar behavioral 


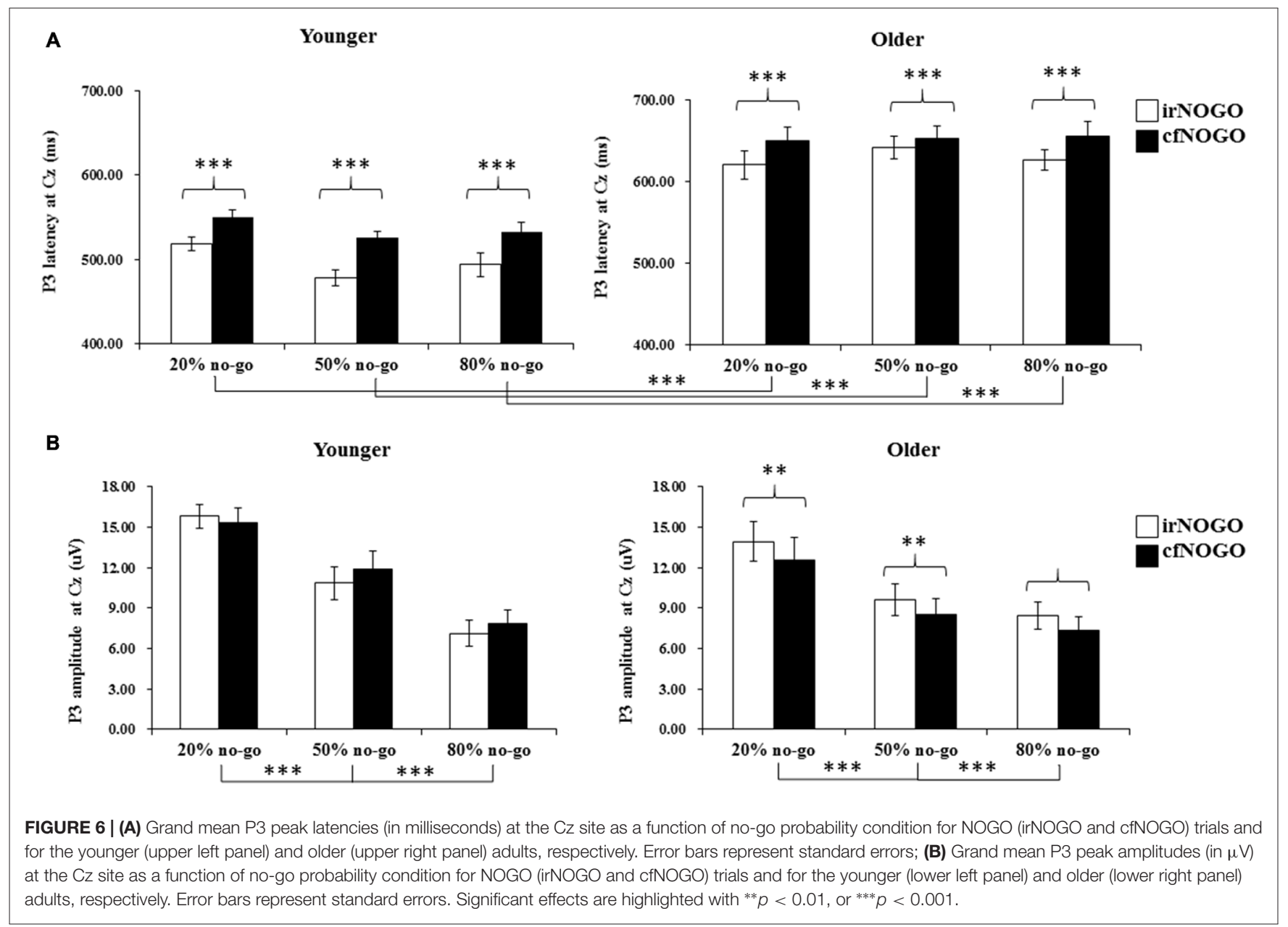

performance on no-go trials (i.e., equivalent commission errors) as compared to the younger adults. These results seemed to suggest that the older adults were capable of inhibiting the no-go trials. In addition, based on the no-go commission error data, it appeared to be more difficult for both younger and older adults to withhold a response to cfNOGO stimuli compared to irNOGO stimuli. Yet, since the behavioral no-go performance can only be measured with commission errors, it is interesting to examine whether or not the underlying neural activity for the no-go trials, as reflected by the ERPs, would be also similar, as shown in the behavioral data between the two age groups.

The current ERP data showed that for the younger adults, the cfNOGO stimuli were associated with smaller N2 amplitudes than those of the irNOGO stimuli, specifically for the $20 \%$

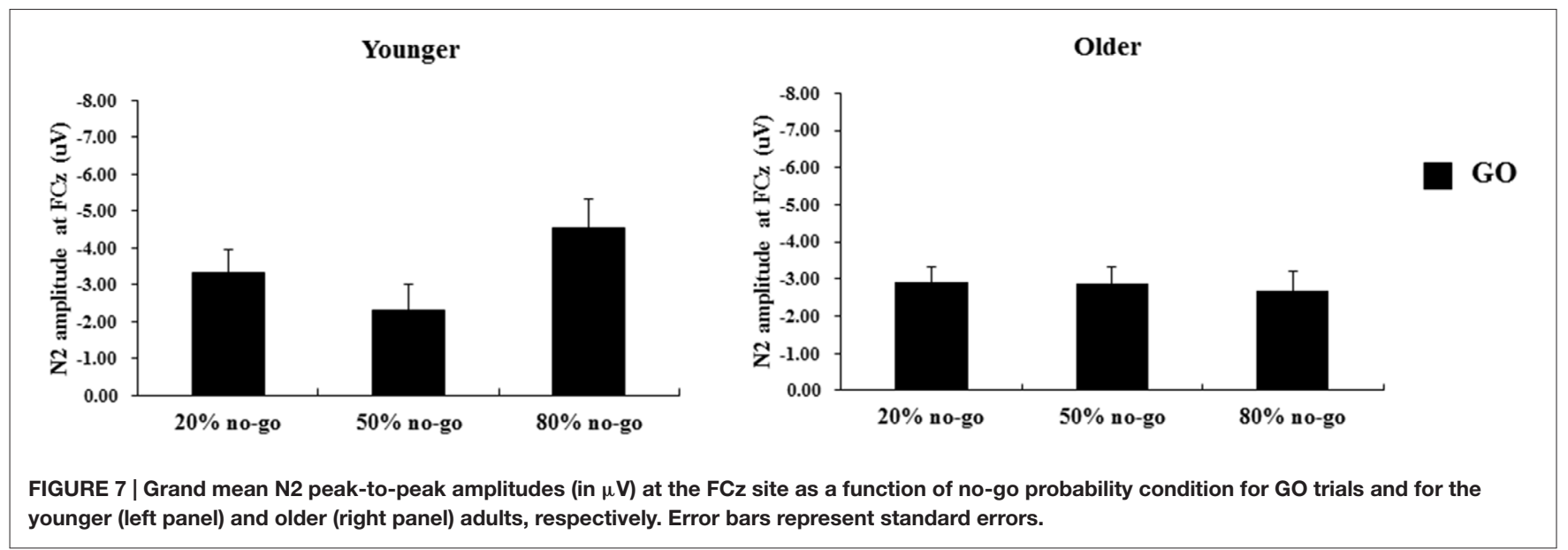




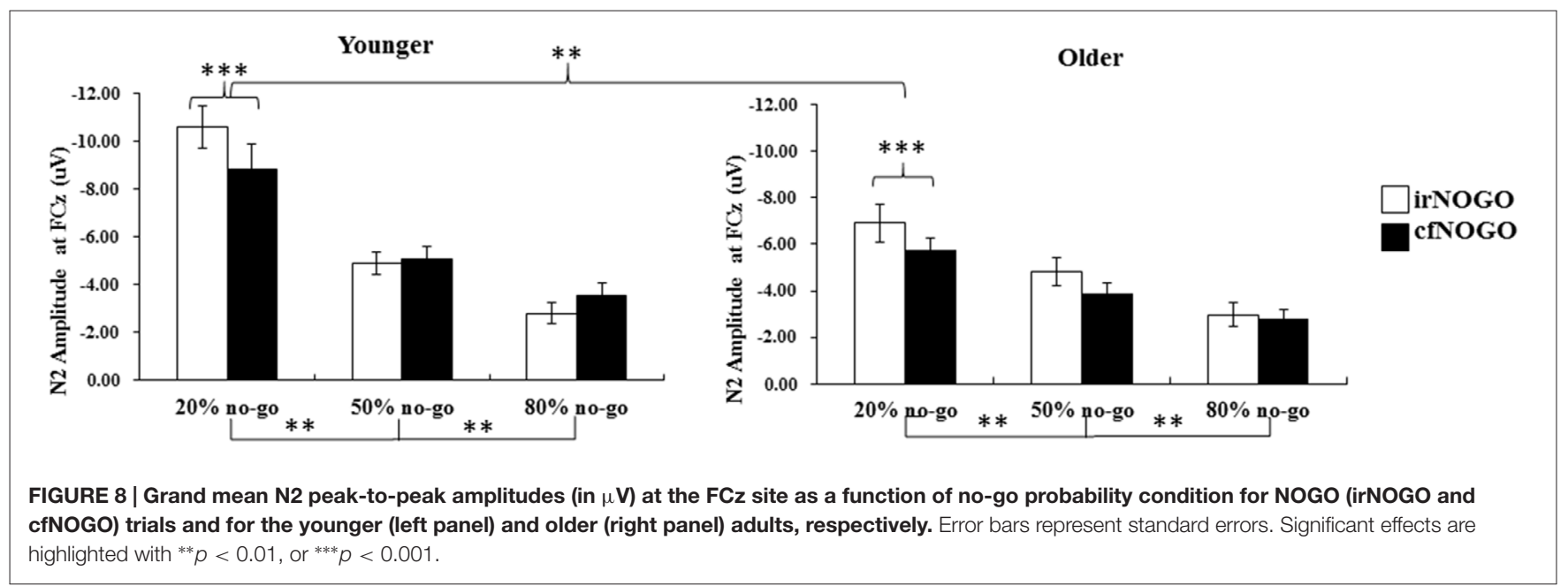

no-go probability condition. Previous research has argued that the N2 enhancement of the no-go trials either reflects the operation of a cognitive top-down inhibition mechanism needed to suppress the incorrect tendency to respond or reflects an electrophysiological correlate of conflict between go and no-go response representations that is detected in ACC (Nieuwenhuis et al., 2003). Since the current results showed that the cfNOGO trials were associated with smaller $\mathrm{N} 2$ amplitudes and were accompanied with more commission errors than the irNOGO trials (but only for the $20 \%$ no-go trial condition), this suggests that either the younger adults experienced less conflict and hence recruited less control processes for the cfNOGO trials than irNOGO trials based on the conflict hypothesis of $\mathrm{N} 2$ (Falkenstein et al., 2001; Van Veen and Carter, 2002a,b) or they were less able to inhibit the cfNOGO stimuli based on the inhibition account (Roche et al., 2005). Nevertheless, since we additionally observed that N2 peak-to-peak amplitudes were also modulated by the no-go stimulus probability for the younger adults, the results appeared to be more consistent with the results reported by Nieuwenhuis et al. (2003); see also Smith et al. (2008), who suggested that $\mathrm{N} 2$ may reflect response conflict rather than inhibition per se.

More importantly, in the current study, the older adults paradoxically exhibited larger P3 amplitudes for the irNOGO than cfNOGO trials, whereas behaviorally they committed more errors in the cfNOGO than irNOGO trials. This seems to suggest that the older adults recruited more control processes in order to conquer the commitment of responses for the no-go trials, especially for the irNOGO trials. The more direct evidence that supports this speculation comes from the correlational analysis between the behavioral performance and P3 amplitudes. The correlation was significant $(r=0.51, p<0.001$; see Figure 9) and showed that better performance (i.e., lower PE) inhibiting the no-go trials was associated with larger the P3 amplitudes in the no-go trials, relative to go trials. This finding was consistent with Vallesi et al. (2009), who showed that the irNOGO trials seemed to be more distracting (and hence cause a larger conflict N2) for the older adults to withhold the response; hence, the older adults needed to recruit more control processes in withholding the response towards the irNOGO trials than the cfNOGO trials, as reflected by their larger no-go P3 peak amplitudes for the irNOGO than cfNOGO trials. However, no such phenomenon was found for the younger adults. This agerelated compensatory response (i.e., conquering the irNOGO stimuli with the price of poorer performance of the cfNOGO stimuli) were specifically seen in the $20 \%$ no-go trial probability condition. The manipulation of the low probability of no-go trials has proved successful in activating frontal lobe structures associated with executive control in other response inhibition tasks (MacDonald et al., 2000). Hence, in this scenario, the older adults might thus recruit more compensatory responses in withholding the responses towards the no-go trials. This might also explain why there was no age effect seen in the behavioral no-go commission errors due to the compensatory responses.

The current finding that the older adults were more distracted by the irNOGO stimuli than the cfNOGO stimuli appears to be consistent with previous reports in the memory research domain, which suggests that older adults (more pronounced after age 65) suffer from increasing vulnerability to internal distraction and weakening concentration skills due to subtle changes in brain activity (Grady et al., 2006). Grady et al. (2006) found that older adults have difficulty activating brain regions necessary for concentration (e.g., reading) and de-activating or tuning down other regions that are associated with internal thoughts (e.g., thinking about yourself, what you did yesterday). Two key regions of the brain that allow the mind to focus on a single task and tune out unwanted thoughts get out of kilter much earlier in life than previously suspected. When the mind pays strict attention, special neural circuits in the prefrontal cortex become more active, and at the same time, related brain areas in the medial frontal lobe (monitoring more general background activity) slack off. Conversely, when the mind is at rest, the level of brain activity in these two regions is then reversed. Such a seesaw activity pattern can begin to break down during memory tasks starting at about age 40 . 


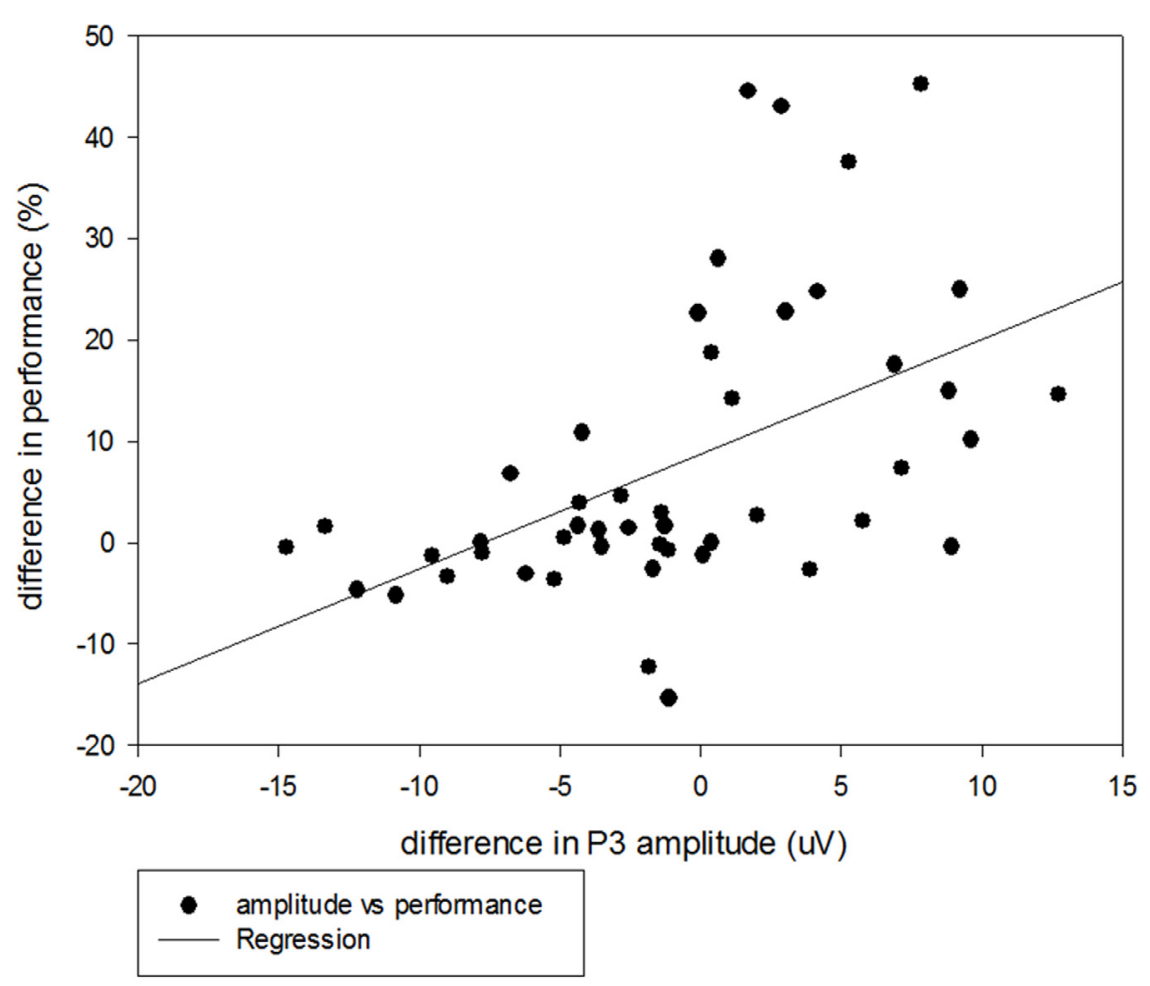

FIGURE 9 | Scatterplot with regression line illustrating the correlation between P3 amplitudes (uV) and behavioral performance (\%) for the irNOGO trials relative to GO trials across all three no-go probability conditions for the older adults.

Another interesting finding of this study is that older adults seemed to devote more effort towards no-go trials, since the nogo probability effect was only seen significantly for the no-go trials, whereas the effect of no-go probability was significant for both the go and no-go trials for the younger adults. While the younger adults exhibited the conventional larger P3 amplitudes on go trials in the low go-prepotency condition than in the high go-prepotency condition, the older adults did not show such a pattern. It is therefore not surprising to observe a significant age effect on the go P3 amplitudes in the 50 and $80 \%$ no-go probability conditions rather than in the $20 \%$ no-go probability condition $(p=0.24)$. On the other hand, the amplitudes of both $\mathrm{N} 2$ and P3 on the no-go trials were found to vary significantly as a function of the relative frequency of no-go probability $(20,50$ and $80 \%$ ) conditions for both age groups. These results suggest that the older adults (similarly to the younger adults) when withholding the responses could be influenced by the no-go trials' probability. Given that the older adults were not influenced by the go trials' probability when executing the responses for the go trials but that they were influenced by the no-go trials' probability when withholding the responses on no-go trials, we could suspect that the older adults seemed to devote more effort to the no-go trials since it was more effortful for them to withhold the responses; hence, they were more sensitive to the no-go trials' probability.

Some final issues should be noted. First, this study used a between-subjects design for the factor of no-go probability which might underestimate the effect of no-go probability on the current results, and therefore the current interpretations regarding the interactions with the no-go probability should be treated with caution. Second, although the MMSE scores for the elderly participants in the current study were all within the normal range (on average 26-28), one may be still concerned that these elderly might exhibit an early sign of mild cognitive impairment. Nevertheless, since we have run ANCOVAs using MMSE as a covariate factor and the result patterns remained the same as reported here, we believe that although the older adults exhibited lower MMSE scores, they did not confound the current findings. Third, the topography of the no-go P3 in the older adults (Figure 4B) seems to be more frontally distributed compared with the younger adults who showed a more central distribution (Figure 3B). This might be a hint toward a posterioranterior shift in aging (PASA, see Davis et al., 2008). In order to examine if the current findings were consistent with the theory of PASA, we re-ran ANOVAs for the P3 data with an additional factor of electrode sites of $\mathrm{FCz}, \mathrm{Fz}$, and $\mathrm{Cz}$. The results of the 4-way ANOVAs showed no significant interactions of electrode sites with all other factors, except its own significant effect; hence, the current data did not seem to show a PASA effect for the elderly. Finally, it has been reported in previous studies that older adults showed similar (Falkenstein et al., 2002) and sometimes stronger no-go P3 amplitudes (Hong et al., 2014) compared with younger adults. However, in this study, it seems that older adults showed smaller no-go P3 amplitudes 
(Figure 6B). Yet, it is important to note that Falkenstein et al. (2002) and Hong et al. (2014) used the P3 difference wave (P3d: no-go minus go ERPs) rather than the absolute no-go P3 amplitude, as reported in this study. When we subtracted go ERPs from no-go ERPs, we likewise observed similar P3d amplitudes (in 20\% condition) and larger P3d amplitudes (in both 50 and $80 \%$ conditions) for the older adults compared with the younger adults. We decided to report go and no-go ERPs separately because of the following reasons: (1) go and no-go trials involve different processes with respect to motor execution and (2) we were more interested in contrasting irNOGO and cfNOGO trials.

To conclude, using ERP data the current study revealed that older adults were more prone to distraction induced by irNOGO stimuli, and therefore they exerted more control processes to conquer such distraction. Furthermore, older adults tended to devote more effort to withholding responses towards the no-go trials, especially in the condition where more control demand was needed (e.g., in the $20 \%$ no-go trials' probability in this study). This study provided a deeper understanding into how older adults adopted strategies in performing the go/no-go task (e.g., devoting attention to no-go trials in order to cope with their deficient inhibition ability). This interpretation appears to be contradictory to the well-known aging hypothesis of inhibition deficits (Hasher and Zacks, 1988). Yet, given that previous research has already demonstrated that older adults would develop a strategy to cope with some task scenarios, we suggest in the current study that older adults performing the

\section{REFERENCES}

Bokura, H., Yamaguchi, S., Matsubara, M., and Kobayashi, S. (2002). Frontal lobe contribution to response inhibition process-an ERP study and aging effect. Int. Congr. Ser. 1232, 17-20. doi: 10.1016/s0531-5131(01) 00677-x

Bruin, K., and Wijers, A. (2002). Inhibition, response mode and stimulus probability: a comparative event-related potential study. Clin. Neurophysiol. 113, 1172-1182. doi: 10.1016/s1388-2457(02)00141-4

Cabeza, R., Anderson, N. D., Locantore, J. K., and McIntosh, A. R. (2002). Aging gracefully: compensatory brain activity in high-performing older adults. Neuroimage 17, 1394-1402. doi: 10.1006/nimg.2002.1280

Davis, S. W., Dennis, N. A., Daselaar, S. M., Fleck, M. S., and Cabeza, R. (2008). Que PASA? The posterior-anterior shift in aging. Cereb. Cortex 18, 1201-1209. doi: 10.1093/cercor/bhm155

Dempster, F. N. (1992). The rise and fall of the inhibitory mechanism: toward a unified theory of cognitive development and aging. Dev. Rev. 12, 45-75. doi: 10. 1016/0273-2297(92)90003-k

Dennis, N. A., and Cabeza, R. (2008). "Neuroimaging of healthy cognitive aging," in The Handbook of Aging and Cognition, Vol. 3, eds F. I. M. Craik and T. A. Salthouse (Mahwah, NJ: Lawrence Erlbaum Associates), 1-54.

Donchin, E., and Coles, M. G. (1988). Is the P300 component a manifestation of context updating? Behav. Brain Sci. 11, 357-374. doi: 10. 1017/s0140525x00058027

Falkenstein, M., Hoormann, J., and Hohnsbein, J. (2001). Changes of error-related ERPs with age. Exp. Brain Res. 138, 258-262. doi: 10.1007/s002210100712

Falkenstein, M., Hoormann, J., and Hohnsbein, J. (2002). Inhibition-related ERP components: variation with modality, age and time-on-task. J. Psychophysiol. 16, 167-175. doi: 10.1027/0269-8803.16.3.167

Fallgatter, A. J., and Strik, W. K. (1999). The NoGo-anteriorization as a neurophysiological standard-index for cognitive response control. Int. J. Psychophysiol. 32, 233-238. doi: 10.1016/s0167-8760(99)00018-5 go/no-go task adopted a compensating strategy of paying more attention to the no-go trials.

\section{ETHICS STATEMENT}

The study was approved by the Ethical committee of the National Cheng Kung University in accordance with the Declaration of Helsinki. Written informed consent was obtained from all subjects before the experiment.

\section{AUTHOR CONTRIBUTIONS}

$\mathrm{SH}$ designed the experiment, wrote the grant proposal, guided in executing the experiment, interpreted the data, and wrote the manuscript; MU analyzed the data, plotted the figures; C-HT conducted the experiment and collected the data.

\section{ACKNOWLEDGMENTS}

We would like to thank the Ministry of Science Technology (MOST) of the Republic of China, Taiwan for financially supporting this research (Contract No. 101-2410-H-006046-MY3 and No. 104-2410-H-006-021-MY2). In addition, this research was, in part, supported by the Ministry of Education, Taiwan, R.O.C., The Aim for the Top University Project to the National Cheng Kung University (NCKU). We also wish to thank American Manuscript Editors (AmericanManuscriptEditors.com) for English proofreading.

Folstein, M. F., Folstein, S. E., and McHugh, P. R. (1975). "Mini-mental state": a practical method for grading the cognitive state of patients for the clinician. J. Psychiatr. Res. 12, 189-198. doi: 10.1016/0022-3956(75) 90026-6

Ford, J. M., Gray, M., Whitfield, S. L., Turken, U., Glover, G., Faustman, W. O., et al. (2004). Acquiring and inhibiting prepotent responses in schizophrenia: event-related brain potentials and functional magnetic resonanceimaging. Arch. Gen. Psychiatry 61, 119-129. doi: 10.1001/archpsyc. 61.2 .119

Grady, C. L., Springer, M. V., Hongwanishkul, D., McIntosh, A. R., and Winocur, G. (2006). Age-related changes in brain activity across the adult lifespan. J. Cogn. Neurosci. 18, 227-241. doi: 10.1162/jocn.2006.18.2.227

Hasher, L., and Zacks, R. T. (1988). Working memory, comprehension and aging: a review and new view. Psychol. Learn. Motiv. 30, 193-225. doi: 10.1016/s00797421(08)60041-9

Hong, X., Sun, J., Bengson, J. J., and Tong, S. (2014). Age-related spatiotemporal reorganization during response inhibition. Int. J. Psychophysiol. 93, 371-380. doi: 10.1016/j.ijpsycho.2014.05.013

Howell, D. C. (2010). Statistical Methods for Psychology. 7th Edn. Belmont, CA: Thomson Wadsworth.

Hsieh, S., and Fang, W. (2012). Elderly adults through compensatory responses can be just as capable as young adults in inhibiting the flanker influence. Biol. Psychol. 90, 113-126. doi: 10.1016/j.biopsycho.2012.03.006

Hsieh, S., and Lin, Y.-C. (2014). The boundary condition for observing compensatory responses by the elderly in a flanker-task paradigm. Biol. Psychol. 103, 69-82. doi: 10.1016/j.biopsycho.2014.08.008

Hsieh, S., Liang, Y.-C., and Tsai, Y.-C. (2012). Do age-related changes contribute to the flanker effect? Clin. Neurophysiol. 123, 960-972. doi: 10.1016/j.clinph. 2011.09.013

Lucci, G., Berchicci, M., Spinelli, D., Taddei, F., and Di Russo, F. (2013). The effects of aging on conflict detection. PLoS One 8:e56566. doi: 10.1371/journal.pone. 0056566 
MacDonald, A. W., III, Cohen, J. D., Stenger, V. A., and Carter, C. S. (2000). Dissociating the role of the dorsolateral prefrontal and anterior cingulate cortex in cognitive control. Science 288, 1835-1838. doi: 10.1126/science.288.5472. 1835

Nieuwenhuis, S., Yeung, N., van den Wildenberg, W., and Ridderinkhof, K. R. (2003). Electrophysiological correlates of anterior cingulate function in a go/no-go task: effects of response conflict and trial type frequency. Cogn. Affect. Behav. Neurosci. 3, 17-26. doi: 10.3758/cabn.3.1.17

Phillips, L. H., and Andrés, P. (2010). The cognitive neuroscience of aging: new findings on compensation and connectivity. Cortex 46, 421-424. doi: 10.1016/j. cortex.2010.01.005

Pires, L., Leitão, J., Guerrini, C., and Simões, M. R. (2014). Event-related brain potentials in the study of inhibition: cognitive control, source localization and age-related modulations. Neuropsychol. Rev. 24, 461-490. doi: 10.1007/s11065014-9275-4

Raz, N. (2000). "Aging of the brain and its impact on cognitive performance: integration of structural and functional findings," in Handbook of Aging and Cognition - II, eds F. I. M. Craik and T. A. Salthouse (Mahwah, NJ: Lawrence Erlbaum Associates Publishers), 1-90.

Roberts, L. E., Rau, H., Lutzenberger, W., and Birbaumer, N. (1994). Mapping P300 waves onto inhibition: Go/No Go discrimination. Electroencephalogr. Clin. Neurophysiol. 92, 44-55. doi: 10.1016/0168-5597(94) 90006-x

Roche, R. A., Garavan, H., Foxe, J. J., and O’Mara, S. M. (2005). Individual differences discriminate event-related potentials but not performance during response inhibition. Exp. Brain Res. 160, 60-70. doi: 10.1007/s00221-0041985-z

Rogers, R. D., Sahakian, B. J., Hodges, J. R., Polkey, C. E., Kennard, C., and Robbins, T. W. (1998). Dissociating executive mechanisms of task control following frontal lobe damage and Parkinson's disease. Brain 121, 815-842. doi: 10.1093/brain/121.5.815

Smith, J. L., Johnstone, S. J., and Barry, R. J. (2008). Movement-related potentials in the Go/NoGo task: the P3 reflects both cognitive and motor inhibition. Clin. Neurophysiol. 119, 704-714. doi: 10.1016/j.clinph.2007.11.042
Tekok-Kilic, A., Shucard, J. L., and Shucard, D. W. (2001). Stimulus modality and Go/NoGo effects on P3 during parallel visual and auditory continuous performance tasks. Psychophysiology 38, 578-589. doi: 10 . $1017 / \mathrm{s} 0048577201991279$

Vallesi, A. (2011). Targets and non-targets in the aging brain: a go/nogo eventrelated potential study. Neurosci. Lett. 487, 313-317. doi: 10.1016/j.neulet.2010. 10.046

Vallesi, A., Stuss, D. T., McIntosh, A. R., and Picton, T. W. (2009). Age-related differences in processing irrelevant information: evidence from event-related potentials. Neuropsychologia 47, 577-586. doi: 10.1016/j.neuropsychologia. 2008.10.018

Van Boxtel, G. J. M., Van der Molen, M. W., Jennings, J. R., and Brunia, C. H. M. (2001). A psychophysiological analysis of inhibitory motor control in the stop-signal paradigm. Biol. Psychol. 58, 229-262. doi: 10.1016/s0301-0511(01) 00117-x

Van Veen, V., and Carter, C. S. (2002a). The anterior cingulate as a conflict monitor: fMRI and ERP studies. Physiol. Behav. 77, 477-482. doi: 10 1016/s0031-9384(02)00930-7

Van Veen, V., and Carter, C. S. (2002b). The timing of action-monitoring processes in the anterior cingulate cortex. J. Cogn. Neurosci. 14, 593-602. doi: 10.1162/08989290260045837

West, R. (2000). In defense of the frontal lobe hypothesis of cognitive aging. J. Int. Neuropsychol. Soc. 6, 727-729. doi: 10.1017/s1355617700666109

Conflict of Interest Statement: The authors declare that the research was conducted in the absence of any commercial or financial relationships that could be construed as a potential conflict of interest.

Copyright (c) 2016 Hsieh, Wu and Tang. This is an open-access article distributed under the terms of the Creative Commons Attribution License (CC BY). The use, distribution and reproduction in other forums is permitted, provided the original author(s) or licensor are credited and that the original publication in this journal is cited, in accordance with accepted academic practice. No use, distribution or reproduction is permitted which does not comply with these terms. 